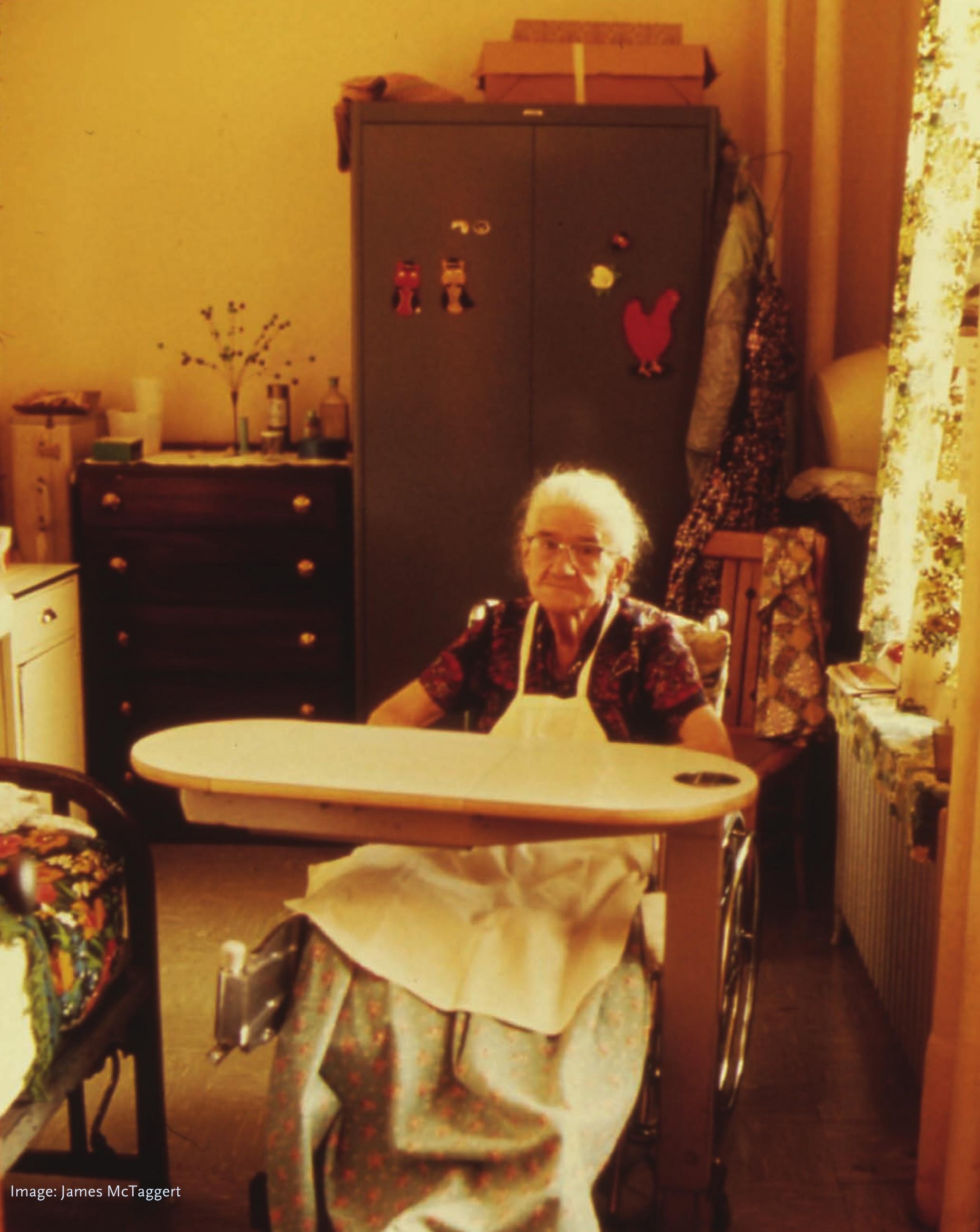




\begin{tabular}{ll}
\hline & $\square$ \\
Nicolas Schönfeld, & Lungenklinik Heckeshorn, \\
Torsten Blum & $\begin{array}{l}\text { HELIOS Klinikum Emil von } \\
\text { Behring, Berlin, Germany }\end{array}$
\end{tabular}

(n)

\section{Palliative care in thoracic oncology}

The vast majority of patients suffering from lung cancer are bound to die from their disease. Correspondingly, chronic or acute symptoms most often dominate the course of their disease from diagnosis to death, and are either caused by the disease itself or, sometimes, by tumourspecific therapeutic measures. Among those symptoms, pain, dyspnoea, fatigue and cough are the most frequent $[1,2]$.

In some European countries, lung cancer patients are mainly treated by pneumologists, including chemotherapy, or in specialised institutions (chest hospitals), which include thoracic surgery and radiotherapy units [3]. This is likely to be mainly due to the fact that frequent local complications caused by lung cancer, such as central airways obstruction, pleural effusion, pneumonia or haemoptysis, all of which require an immediate pneumological management. Consequently, pneumologists began to meet the upcoming challenge and need for palliative treatment of their patients with thoracic malignancies, as well as pneumological patients with non-malignant terminal illnesses accompanied by a high burden of symptoms [4].

In recent years, palliative care has changed from being an additional provision to tumourspecific treatment measures limited to end-oflife situations. Nowadays increasingly it has developed to being the most comprehensive treatment concept, at least for patients with incurable disease. Previously, pneumologists' understanding of palliative care was more or less restricted to the medical treatment of disease-related symptoms [5]. Even now, such a restricted conceptual scope is perceived by some authors [6]. However, it is becoming more evident that palliative care has the potential to be the most individual, complex and thorough approach to any chronically ill patients, especially those with malignancy, who need more than medical treatment, as well as prognostically relevant or curative treatment methods specific to the tumour. This widening approach is given in table 1 [7].

The growing perception of palliative care by organ-specific experts is, on the one hand, determined by the original concept of the founder of the discipline, Dame Cicely Saunders (1918-2005). Based upon her fourfold understanding of the suffering individual and their "total pain", consisting of the patient's physical as well as psychological, social and spiritual pain without exclusion (fig. 1) [8]. It is remarkable that the term "holistic medicine" is usually applied to more general "alternative" or "complementary" medical approaches, but not used by palliative care professionals for their work. In the modern understanding of palliation the expression "holistic" would appear most appropriate, as palliation requires much more than medicine, namely a manifold care to truly address the whole person, including their social network [9].

On the other hand, pneumologists and thoracic surgeons have learned that, even
Statement of Interest None declared.

HERMES syllabus link: modules: B.2, E.1.13, F.8, I.6 
Table 1. WHO definition of palliative care [7]:

Palliative care is an approach that improves the quality of life of patients and their families facing the problem associated with lifethreatening illness, through the prevention and relief of suffering by means of early identification and impeccable assessment and treatment of pain and other problems, physical, psychosocial and spiritual. Palliative care:

- provides relief from pain and other distressing symptoms

- affirms life and regards dying as a normal process

- intends neither to hasten nor postpone death

- integrates the psychological and spiritual aspects of patient care

- offers a support system to help patients live as actively as possible until death

- offers a support system to help the family cope during the patients illness and in their own bereavement

- uses a team approach to address the needs of patients and their families, including bereavement counselling, if indicated

- will enhance quality of life, and may also positively influence the course of illness

- is applicable early in the course of illness, in conjunction with other therapies that are intended to prolong life, such as chemotherapy or radiation therapy, and includes those investigations needed to better understand and manage distressing clinical complications

after successful curative treatment measures, patients' preoperative loss of quality of life frequently persists after surgery, as expressed by continuously impaired physical function, loss of physical and emotional roles, reduced energy and lowered mental status [10]. Most recently, the prospective comparison between patients receiving early palliative care integrated with standard oncology care versus standard oncology care with palliative care given only on request, revealed that early palliative care may contribute to a significant increase in life expectancy [11]. These two facts do not seem to fit into our previous conventional understanding of tumour-specific treatment which still dominates our scientific world of thought during scientific conferences. Thus, are we, as pneumologists, prepared to understand and accept the obviously fundamental interrelations of medical findings, symptoms, patients' subjective needs and diverse influences of care on our patients' lives?

\section{Assessment of patients' distress}

Before addressing the treatment options for various causes and aspects of what comprises the term "total pain", those who take care of the patient have to become aware of the patient's condition, both overall and in detail. Self-reporting by the patient is still the cornerstone of assessment; nevertheless, the time and character of the patient's contact with professionals and the communicative method is crucial for a successful therapeutic concept and its evaluation [5].

A well-evaluated tool is the distress thermometer (fig. 2) [12]. Scale and image 
help to express the degree of symptom load, a comprehensive list of items should describe the single or combined dimensions of discomfort. The timeline of a day or other time period may influence the result of assessment considerably; the same is true for the situation, i.e. pain may be a minor problem at rest, whereas standing up can be an insuperable problem. Most of the patients with lung cancer experience more than one modality of discomfort [2].

\section{Physical discomfort}

\section{Physical pain}

The medical basis of pain management has been well described, and the available pharmaceutical and radiotherapeutic measures should be sufficient to achieve a substantial, and preferably complete, pain relief for virtually each patient $[5,13,14]$. A guideline should always be the basis for the treatment concept that has to be individualised. Simplicity and effectiveness should determine the treatment programme. The pharmaceutical choice offers widely available non-steroidal anti-inflammatory and analgetic drugs, opioids and bisphosphonates, the latter specifically directed to bone metastases, but not to tumour cells. Adjunct drugs, such as antidepressants or neuroleptic substances may support the analgetic effect [5]. Radiotherapy for local pain is highly effective but requires a radiotherapy unit. The same is true for the application of radiopharmaceuticals [15]. Nerve blocks or surgical interventions for painful lesions can be offered after interdisciplinary consideration [5]. Physical modalities can be especially effective if tension or immobilisation aggravate the symptoms.

\section{Dyspnoea}

Patients with thoracic malignancies show a distinctly high prevalence of dyspnoea when compared that have patients with other kinds of malignancies [16]. Treatment options for dyspnoea are as widespread as the causes of dyspnoea. Central airway obstruction by the tumour, i.e. in the trachea, main stem bronchi or in the bronchus intermedius, can be relieved by endoscopic techniques [6]. Mechanical or thermal debulking, stenting or brachytherapy are the main procedures that are frequently applied on the basis of experience, rather than evidence. Nevertheless, tracheal obstruction

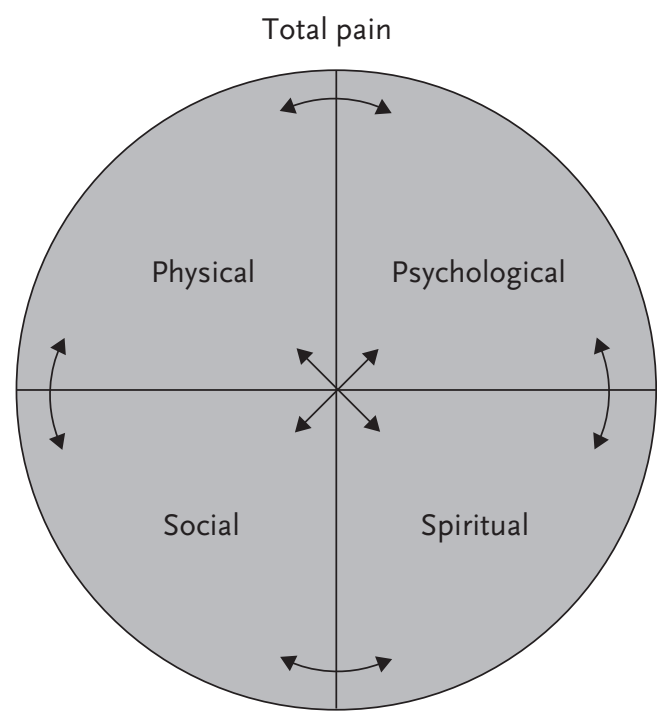

Figure 1

The total pain experience: an interactive model.

can be considered an emergency that should be relieved if the patient is willing to undergo the procedure.

In cases of symptomatic pleural effusion, the pneumologists are better off: the least invasive stepwise approaches range from simple thoracentesis, indwelling catheters, talc poudrage during medical thoracoscopy to surgical measures [17]. The latter procedure, mostly performed using video-assisted thoracic surgery (VATS), was identified to be the most invasive approaches with a potential for procedure-related morbidity. Thus, the joint European Respiratory Society (ERS) and European Society of Thoracic Surgeons (ESTS) task force on malignant pleural mesothelioma clearly decided to recommend talc poudrage as the method of choice for symptomatic effusion and not palliative pleural debulking, which was widely performed for decades, without any published evidence to support it [18]. A similar situation could probably be stated for secondary pleural malignancy with effusion. As VATS is the most invasive of all the available procedures, it should be indicated with caution as medical thoracoscopy with talc poudrage and drainage is far less invasive [17].

For all kinds of invasive measures, the indication depends on the remaining life expectancy of the patient. The shorter the life expectancy, the less invasive all measures should be. But where are the lines? The strongest requirements for a multi-site communication, with the patient at the centre of the 


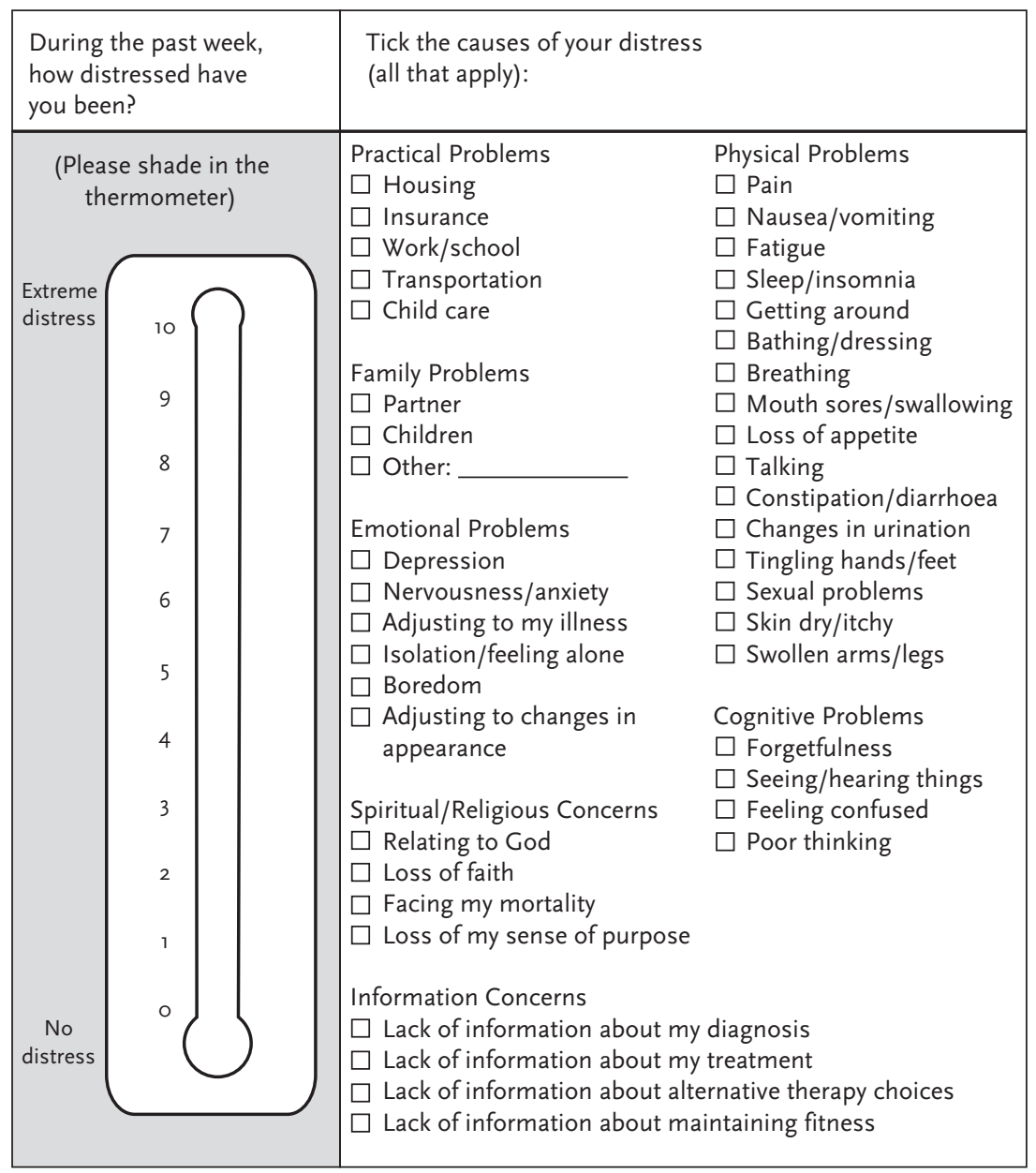

Figure 2

The distress thermometer. Reproduced with permission from the NCCN Clinical Practice Guidelines in Oncology (NCCN Guidelines ${ }^{\circledR}$ ) for Distress Management V.2.2013. (C) 2012 National Comprehensive Cancer Network, Inc. All rights reserved. The NCCN Guidelines ${ }^{\circledR}$ and illustrations herein may not be reproduced in any form for any purpose without the express written permission of the NCCN. To view the most recent and complete version of the NCCN Guidelines, go online to NCCN.org. NATIONAL COMPREHENSIVE CANCER NETWORK ${ }^{\circledR}$, NCCN ${ }^{\circledR}, N C C N$ GUIDELINES ${ }^{\circledR}$, and all other NCCN Content are trademarks owned by the National Comprehensive Cancer Network, Inc.

discussion, are given for intensive care measures to relieve dyspnoea. Non-invasive ventilation has been identified as a tool for mere palliation, but requires an interdisciplinary recommendation and a full and conscious consent of the patient [19]. Therefore, the information given to the patient and the discussion should take place before the emergency, at an earlier point of the patient's disease. However, if prepared, intensive care units should be open for lung cancer patients. From openly aware situations like these, we can conclude that, at least in part, consideration of any possibly invasive treatment in accordance with the patient can take place in the status of patient autonomy and relative well-being for the individual patient. A preventive power of attorney should, whenever possible, accompany the declaration of the patient's treatment requirements concerning themselves.

A non-invasive treatment of dyspnoea is offered by opioids, alongside oxygen insufflation in case of hypoxia. The anxiety, which invariably goes with stronger dyspnoea, can be treated effectively with low doses of morphine or other substances. Therefore, opioids are regularly part of guidelines for palliation of thoracic oncological patients with dyspnoea [5], and an alternative for patients who do not respond adequately to opioids may be inhaled furosemide [2]. 


\section{Cachexia}

Loss of appetite, dysphagia, early satiety or changes in taste may contribute to weight loss, as much as depression or chronic inflammation can, in lung cancer patients. Besides diet counselling, pharmaceuticals are available to stimulate appetite and gain of weight (megestrol actetate, tetrahydrocannabinol or olanzapine) $[2,9,11]$. If the appetite improves, quality of life usually also improves. Antiemetics may be especially helpful in cases of treatment-related nausea. This problem is often disregarded, as it appears to be limited to just a few days after chemotherapy or radiotherapy treatment. Yet even short-term weight loss can be substantial and long-standing and should, whenever possible, be avoided by early intervention.

\section{Fatigue}

Fatigue as a result of the aforementioned conditions or a self-standing problem is experienced by $>80 \%$ of lung cancer patients and, for at least one-third, severely [2]. Furthermore, anaemia or side effects of chemotherapy, radiotherapy or opioids may induce weakness. A specific mechanism could be a mismatch between perception and activation of muscles in the central nervous system. There is no specific treatment available for the symptom of fatigue. Careful evaluation of each patient should direct treatment concepts to the assumed underlying conditions. Fatigue is treatable by psychoeducational interventions for self-management [9].

\section{Haemoptysis}

Similar to central airway obstruction, haemoptysis is a condition that can be lifethreatening and is usually accompanied by anxiety. Interventional procedures are available to stop bleeding and remove blood from central airways (argon plasma coagulation, Nd YAG photocoagulation, mechanical recanalisation during rigid bronchoscopy, balloon tamponade, etc.) [6]. Peripheral bleeding lung tumours may give cause for palliative resection. Bronchial artery embolisation is another option with only temporary chances of success. Yet all of these interventions should be indicated with respect to the overall prognosis and the previous course of treatment. In cases of life-threatening haemoptysis, sedation and care for the dying individual may be the only adequate approach.

\section{Cough}

Cough is one of the most frequent symptoms and may be quite painful for the patient, especially in instances of repeated and neverending blows [20]. The impact of permanent cough on quality of life domains, such as social, psychological or physical is significant. Therefore, the problem should not be underestimated, despite the regrettable fact that current methods for the palliation of cough are lagging behind the management of other cancer symptoms. Beyond the prescription of common antitussives, determinable reasons for cough should be identified, such as endobronchial manifestation of the disease, concomitant COPD with or without infection, or pleural effusion. Specific treatment of underlying conditions, if available, provide the best chance for relief.

\section{Psychosocial discomfort}

Shortly after diagnosis and at the end of treatment, patients with lung cancer are highly susceptible to develop a clinically relevant depression [21]. Further situations that can result in psychological deterioration are disease progression and the terminal stage. Lower education status, female sex, older age and poor performance status are additional risk factors for psychosocial discomfort. Social rejection or isolation, financial insecurity or internalised shame are frequent triggers of depression. Some lung cancer patients may misperceive that they are stigmatised by their disease, which has a similar effect to an actual stigmatisation.

Thus, psychotherapeutic support should be offered to all lung cancer patients trying to alleviate or prevent manifest depression and its associated symptoms [5]. Medical treatment of manifest depression can be indicated as an adjunct to psychosocial care. Social support is able to strengthen the autonomy of the patient facing complex treatment options. Care planning in advance can be enabled by skilled discussants, who clearly communicate what medical treatments are available and elicit patients' values and emotions, especially considering physician-patient communication is frequently limited or even inadequate [22]. The same is true for relatives, especially in the case of loss of orientation of the patient themself. The coordination of services is another important contribution undertaken by social workers, particularly in home-care settings. 


\section{Spiritual needs}

Especially in a secular surrounding or in a situation of individualistic or rudimentary spirituality, religious or spiritual needs of patients can easily be missed or misperceived. Nevertheless, the majority of patients will express signs of fear, doubt or despair, will feel that life is not worthwhile, feel isolated or ask for a meaning of their disease, or say "What have I done to deserve it?" This kind of uncertainty and loss of control are central to the patient's spiritual distress [23].

The recognition of spiritual feelings, questions and needs, by the patient themselves or by relatives or caretakers, probably goes along with the stages of recognition of death and dying. Despite methodological critics in the meantime, we can learn from the Kübler-Ross model that, in those patients suffering from grief, the impulse of hope or resilience is present up to and even in the last stage, which is accepting death, while coping with the situation of life-limiting disease [24].

In such a status of acceptance, the patient often appears devoted to approval, which may not be related solely to the powerlessness of the dying individual. Theologically, this devotion can even in non-religious patients or individuals, respectively, be interpreted as hope against all hope (such as in Romans 4.18). In this context, each person appears to own, in the middle of their existence, an unconditional hope. This hope, as a strong resource, enables a freedom for the individual by which they can leave virtually all goods that they kept hold of as a particular good in what they deemed to be a mundane life, behind. Instead, a patient can be strengthened to entrust themselves and their life to the inconceivable secret which forms an absolute future and which we call God [24].

Not all caretakers will find themselves prepared to offer help to and accompany a patient who indicates spiritual distress on such a path [23]. Therefore, communication trained and skilled persons (e.g. healthcare chaplaincy) should always be integrated in a team of palliative carers.

\section{Organisation of palliative care}

It is believed that lung cancer care in general as well as palliative care can be provided more successfully by a multidisciplinary team $[25,26]$. The wide variety of problems that have been outlined in this article shows the need to be organised in a multiprofession manner, and research seems to confirm this recommended approach. Besides the quality and success of palliation offered to the patient, the care for caregivers who are preoccupied to cope with the burden of grief, death and dying themselves while accompanying the patients and their relatives, requires professional structures such as supervision, as well as psychological support and spiritual assistance.

Inpatients will profit mostly from palliative care units within chest hospitals offering all kinds of interventional and tumour-specific treatment modalities needed for the treatment of patients with thoracic malignancies [3]. For outpatients, a network of caregivers should be ready to cover more than just the absolutely bare minimum care in order to enable the person to die at home, which most of the patients prefer.

\section{Conclusion}

Palliative care, in its comprehensiveness, should be offered as early as possible to patients with newly diagnosed lung cancer and other thoracic malignancies, at least the moment where curative intentions fade. Palliation comprises a wide spectrum of offers to the patient, including medical treatment, nursing, social and psychological support, and pastoral care in an holistic concept, which focuses on patient's symptoms and quality of life. Palliative care is at best offered by a multiprofessional team, and not only to the patient, but also to relatives and other caregivers. The integration of palliative care into other disciplines, such as pneumology in its present key role for the diagnosis and treatment of thoracic malignancies, is a crucial task for the future in order to improve both the quality of life and treatment for the vast majority of patients who cannot expect a cure from tumour-specific treatment concepts. 


\section{References}

1. Freedman OC, Zimmermann C. The role of palliative care in the lung cancer patient: can we improve quality while limiting futile care? Curr Opin Pulm Med 2009; 15: $321-326$.

2. Davis MP. The emerging role of palliative medicine in the treatment of lung cancer. Cleveland Clin J Med 2012; 79: eS51-eS55.

3. Blum T, Schönfeld N, Kollmeier J, et al. Lungenkrebs in Deutschland - zur Lage der Nation, [Lung cancer in Germany - the current state of management.] Pneumologie 2011; 65: 7-18.

4. Heigener DF, Rabe KF. Palliative care concepts in respiratory disease. Respiration 2011; 82: 395-399.

5. Kvale PA, Simoff M, Prakash UBS. Palliative care. Chest 2003; 123: 284S-311S.

6. Gompelmann D, Eberhardt R, Herth FJH. Advanced malignant lung disease: what the specialist can offer. Respiration 2011; 82: 111-123.

7. WHO Definition of Palliative Care. Available from www.who.int/cancer/palliative/definition/en Date last accessed: September 2012.

8. Mehta A, Chan LS. Understanding the concept of "total pain": a prerequisite for pain control.J Hospice Palliative Nursing 2008; 10: 26-32.

9. Rueda JR, Solà I, Pascual A, et al. Non-invasive interventions for improving well-being and quality of life in patients with lung cancer. Cochrane Database Syst Rev 2011; 9: CDoO4282.

10. Handy JR, Asaph JW, Skokan L, et al. What happens to patients undergoing lung cancer surgery? Outcomes and quality of life before and after surgery. Chest 2002; 122: 21-30.

11. Temel JS, Greer JA, Muzikansky A, et al. Early palliative care for patients with metastatic non-smallcell lung cancer. N Engl J Med 2010; 363: 733-742.

12. Graves KD, Arnold SM, Love CL, et al. Distress screening in a multidisciplinary lung cancer clinic: prevalence and predictors of clinically significant distress. Lung Cancer 2007; 55: 215-224.

13. Temel ST, Pirl WF, Lynch TJ. Comprehensive symptom management in patients with advancedstage non-small-cell lung cancer. Clin Lung Cancer 2006; 7: 241-249.
14. Mercadante S, Vitrano V. Pain in patients with lung cancer: pathophysiology and treatment. Lung Cancer 2010; 68: 10-15.

15. Bauman G, Charette M, Reid R, et al.

Radiopharmaceuticals for the palliation of painful bone metastasis - a systematic review. Radiother Oncol 2005; 75: 258-270.

16. Alt-Epping B, Stäritz AE, Simon ST, et al. What is special about patients with lung cancer and pulmonary metastases in palliative care? Results from a nationwide survey.J Palliat Med 2012; 15: DOI: $10.108 \mathrm{~g} / \mathrm{jpm} .2011 .0434$.

17. Antony VB, Loddenkemper R, Astoul P, et al. Management of malignant pleural effusion. Eur Respir J 2001; 18: 402-419.

18. Scherpereel A, Astoul P, Baas P, et al. Guidelines of the European Respiratory Society and the European Society of Thoracic Surgeons for the management of malignant pleural mesothelioma. Eur Respir J 2010; 35: 479-495.

19. Schönfeld N, Timsit JF. Overcoming a stigma: the lung cancer patient in the intensive care unit. Eur Respir J 2008; 31: 3-5.

20. Harle AS, Blackhall FH, Smith JA, et al. Understanding cough and its management in lung cancer. Curr Opin Support Palliat Care 2012; 6: 153-162.

21. Gonzalez BD, Jacobson PB. Depression in lung cancer patients: the role of perceived stigma. PsychoOncology 2012; 21: 239-246.

22. Nelson JE, Gay EB, Berman AR, et al. Patients rate physician communication about lung cancer. Cancer 2011; 117: 5212-5220.

23. Murray SA, Kendall M, Boyd K, et al. Exploring the spiritual needs of people dying of lung cancer or heart failure: a prospective qualitative interview study of patients and their carers. Palliat Med 2004; 18: 39-45.

24. Rahner, Karl. Christians at the crossroads, Burns \& Oates: London, 1975.

25. Ellis PM. The importance of multidisciplinary team management of patients with non-small-cell lung cancer. Curr Oncol 2012; 19: S7-S15.

26. Borneman T, Koczywas M, Cristea M, et al. An interdisciplinary care approach for integration of palliative care in lung cancer. Clin Lung Cancer 2008; 9: 352-360.
All views and opinions expressed in articles published in Breathe are those of the author and do not necessarily represent the views of the Chief Editor or ERS or staff members 